\section{Geringe Feinstaubbelas- tung erhöht das Risiko für Stridor und Asthma}

Bowatte $G$ et al. Traffic-related air pollution exposure over a 5-year period is associated with increased risk of asthma and poor lung function in middle age. Eur Respir J 2017; 50: 1602357. doi:10.1183/13993003.02357-2016

Die australische Arbeitsgruppe hatte in einer Querschnittsstudie bereits gezeigt, dass die durchschnittliche jährliche Feinstaubbelastung das Risiko für Atembeschwerden und Asthma bronchiale erhöhte. Das antioxidative Genprofil modulierte die Wahrscheinlichkeit. Jetzt überprüften die Autoren die langfristigen Auswirkungen der verkehrsassoziierten Exposition (TRAP) auf das Erkrankungsrisiko in Abhängigkeit vom Glutathionmetabolismus.

Der Glutathionstoffwechsel ist entscheidend für die Biotransformation körpereigener/körperfremder Substanzen und schützt den Organismus vor oxidativem Stress. Varianten der Glutathion-S-Transferase-T1 (GSTT1) deoxigenieren reaktive Sauerstoffspezies unterschiedlich effektiv. Null-Carrier waren durch eine TRAP besonders gefährdet. Die Daten für die Studie über die langfristigen TRAP-Auswirkungen stammten aus der TAH-Studie (Tasmanian Longitudinal Health Study) mit Teilnehmern des Geburtsjahrgangs 1961. Insgesamt 709 Einwohner nahmen mit 45 Jahren und 50 Jahren an den Untersuchungen teil. Die Autoren bestimmten

- den Wohnabstand zu einer Hauptstraße $(<200 \mathrm{~m} />200 \mathrm{~m})$,

- die Stickoxidexposition,

- die $\mathrm{FEV}_{1}$ und $\mathrm{FEV}_{1} / \mathrm{FVC}$,

- Atembeschwerden und Asthma im Vorjahr und

- den Genotyp der Glutathion-S-Transferase.

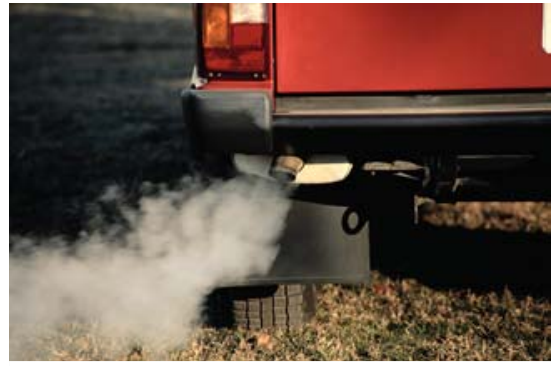

- Auch eine geringe Feinstaubexposition ist mit einem gesteigerten Risiko für Atemwegserkrankungen assoziiert. Bildquelle: PhotoDisc

50-Jährige rauchten seltener, hatten weniger häufig Asthma und nutzten öfter saubere Umweltenergien zum Kochen und Heizen als 45-Jährige. Die Wohnentfernung zu einer Hauptstraße <200m war in beiden Altersgruppen mit einer signifikant höheren Stickoxidbelastung $\left(\mathrm{NO}_{2}\right)$ assoziiert, die mit satellitenbasierten Landnutzungsmodellen ermittelt wurde. Die gesteigerte Exposition und die verkehrsbelastete Wohnsituation erhöhten die Prävalenz von Asthma bronchiale (Odds Ratio OR 1,49; $95 \%$-Konfidenzintervall KI 1,09-2,05 und OR 1,10; $95 \%-K I$ 0,96-1,27). Ähnliche Resultate ergaben sich für den Zusammenhang zwischen Wohnlage und Atembeschwerden ohne Erkältung (OR 1,61; 95\%-KI 1,19-2,19). Die Nähe zu einer Hauptstraße reduzierte das $\mathrm{FEV}_{1}(-0,32)$, die FVC $(-0,20)$ und FEV 1 FVC $(-0,19)$. Die Adjustierung nach Alter, Geschlecht und Körpergröße änderte die Resultate nicht.

Der GSTT1-Polymorphismus modifizierte die Auswirkungen der Feinstaubbelastung auf die Lungenfunktion. Die $\mathrm{NO}_{2}{ }^{-}$ Exposition reduzierte bei 45-50-Jährigen signifikant die Atmungsparameter und erhöhte die Erkrankungswahrscheinlichkeit, wenn sie Null-Carrier für GSTT1 waren (homozygot für das GSTT1 $^{*} 0$ Allel). Träger von GSTP1 val/val und ile/val hatten bei einer hohen $\mathrm{NO}_{2}{ }^{-}$ Belastung eine signifikant gesteigerte Prävalenz für Atembeschwerden und Asthma. Personen mit $\geq 1$ funktionalem GSTM1-Allel hatten ein signifikant reduziertes $\mathrm{FEV}_{1}$, wenn sie nahe einer Hauptstraße lebten.
FAZIT

Die langfristige TRAP-Exposition war mit einem gesteigerten Risiko für Atembeschwerden und Asthma im mittleren Lebensalter assoziiert, wobei das individuelle Genprofil die Effekte modulierte. Die jährliche Feinstaubbelastung von 23,8 und 22,9 ppb war gering und unterschritt die internationalen Grenzwerte. Dass dennoch eine Risikosteigerung bestand, zeigte die Relevanz einer geringen Exposition für die pulmonale Gesundheit. Personen mit einem sensiblen Genstatus waren besonders gefährdet, so die Autoren.

Dr. med. Susanne Krome, Melle 